\title{
Anti-HER2 Bi-specific Monoclonal Antibody ZW25
}

National Cancer Institute

\section{Source}

National Cancer Institute. Anti-HER2 Bi-specific Monoclonal Antibody ZW25. NCI

Thesaurus. Code C130010.

An eng ineered bi-specific monoclonal antibody that targets two different epitopes of the human tumor-associated antigen (TAA) epidermal growth factor receptor 2 (HER2) with potential immunomodulating and antineoplastic activities. After binding to HER2 on the tumor cell surface, anti-HER2 bispecific monoclonal antibody ZW25 induces a cytotoxic T-lymphocyte (CTL) response and antibody-dependent cell-mediated cytotoxicity (ADCC) against tumor cells that overexpress HER2. Antibody-dependent cellular phagocytosis. $(A D C P)$ is also induced and further stimulates the immune system to kill HER2overexpressing tumor cells. In addition, binding of ZW25 to HER2 induces receptor internalization, which inhibits HER2 activation, HER2-mediated signaling and HER2mediated tumor cell growth. HER2, a tyrosine kinase receptor, is overexpressed by many cancer cell types. 